\title{
Hybrid poly(lactic acid)/nanocellulose/nanoclay composites with synergistically enhanced barrier properties and improved thermomechanical resistance
}

Trifol Guzman, Jon; Plackett, David; Sillard, Cecile; Szabo, Peter; Bras, Julien; Daugaard, Anders Egede

Published in:

Polymer International

Link to article, DOI:

10.1002/pi.5154

Publication date:

2016

Document Version

Peer reviewed version

Link back to DTU Orbit

Citation (APA):

Trifol Guzman, J., Plackett, D., Sillard, C., Szabo, P., Bras, J., \& Daugaard, A. E. (2016). Hybrid poly(lactic acid)/nanocellulose/nanoclay composites with synergistically enhanced barrier properties and improved thermomechanical resistance. Polymer International, 65(8), 988-995. https://doi.org/10.1002/pi.5154

\section{General rights}

Copyright and moral rights for the publications made accessible in the public portal are retained by the authors and/or other copyright owners and it is a condition of accessing publications that users recognise and abide by the legal requirements associated with these rights.

- Users may download and print one copy of any publication from the public portal for the purpose of private study or research.

- You may not further distribute the material or use it for any profit-making activity or commercial gain

- You may freely distribute the URL identifying the publication in the public portal 
Hybrid poly(lactic acid)/nanocellulose/nanoclay composites with synergistically enhanced barrier properties and improved thermomechanical resistance

Jon Trifola , David Plackett ${ }^{\mathrm{b}}$, Cecile Sillard ${ }^{\mathrm{c}}$, Peter Szabo ${ }^{\mathrm{a}}$, Julien Bras ${ }^{\mathrm{c}}$, Anders E. Daugaard ${ }^{\mathrm{a}}$

${ }^{a}$ Danish Polymer Centre, Department of Chemical and Biochemical Engineering, Technical University of Denmark, Søltofts Plads, Building 229, DK-2800, Kgs. Lyngby, Denmark

${ }^{\mathrm{b}}$ Faculty of Pharmaceutical Sciences, University of British Columbia, 2405 Wesbrook Mall, Vancouver, BC V6T 1Z3, Canada

${ }^{\text {C } L G P 2 / G r e n o b l e ~ I N P-P a g o r a / C N R S, ~} 461$ rue de la papeterie, Domaine universitaire, C10065, 38402 Saint Martin d'Hères Cedex, France

Keywords:

Cellulose nanofibres (CNF), nanoclay, polylactic acid (PLA), nanocomposites, barrier properties, thermomechanical properties

\section{Abstract:}

Poly(lactic acid) (PLA)-based hybrid nanocomposites (PLA, nanoclay and nanocellulose) were prepared by reinforcing neat PLA with commercially available nanoclay (Cloisite $\mathrm{C} 30 \mathrm{~B}$ ) and nanocellulose, in the form of either partially acetylated cellulose nanofibres (CNF) or nanocrystalline cellulose (CNC). Composites with 1 or 5 wt\% of nanocellulose, in combination with 1, 3 and 5 wt\% of nanoclay, were prepared, and their barrier properties were investigated. It was found that the combination of clay and nanocellulose clearly results in synergistic behaviour in terms of the oxygen transmission rate (OTR) through a reduction of up to $90 \%$ in OTR and a further reduction in the water vapour transmission rate (WVTR) of up to $76 \%$. In addition, the nanocomposite films showed improved thermomechanical resistance and improved crystallisation kinetics while maintaining high film transparency. This makes hybrid PLA/CNF/C30B nanocomposites a very promising material for food packaging applications.

\section{Introduction}


There is a constant interest in finding bio-based materials for food packaging, in order to substitute currently used petrochemical-based polymers such as polyethylene (PE), polypropylene (PP) and poly(ethylene terephthalate) (PET). However, biopolymers are generally considered to have poorer performance than classical petroleum-based polymers. In the specific case of PLA, its brittleness, slow crystallisation rate, poor thermostability and only moderate oxygen and water vapour barrier properties have prevented its use as a packaging material ${ }^{1}$. Accordingly, many different strategies have been investigated to enhance the performance of PLA, including its chemical or physical modification ${ }^{2}$, blending ${ }^{3}$, the use of reinforcing agents such as natural fibres ${ }^{4}$, other kinds of natural fillers $^{5}$ or nanomaterials ${ }^{6,7}$. Among these options, nanocellulose, in the form of either cellulose nanofibres or nanocrystalline cellulose, has been particularly widely used, both as a single reinforcing agent ${ }^{8}$ or as a multilayer structure ${ }^{9}$, not only due to good reinforcing properties ${ }^{10}$, but also due to their inherent biodegradability, bio-based origins and availability.

In our previous work acetylated cellulose nanofibres (CNFs) were prepared through a combination of alkali and acetylation treatments, while nanocrystalline cellulose (CNC) was prepared by acid hydrolysis ${ }^{11}$. The prepared nanocelluloses were compared with commercially available nanoclay (cloisite-C30B) as fillers for PLA-based nanocomposites for food packaging applications. The CNF based composites had significantly improved thermal stability and decreased oxygen transmission rate (OTR) as well as reduced water vapour transmission rate (WVTR) (up to a $64 \%$ decrease in OTR at $23^{\circ} \mathrm{C}$ and $50 \% \mathrm{RH}$ and up to a $46 \%$ of decrease in WVTR). However, these changes are not sufficient when compared with the properties of PET, which is considered the industrial standard in terms of permeability. Indeed, barrier properties are critical for food packaging applications, since oxygen and water can augment microbiological activity, which in turn will boost food degradation. PET has an OTR of approximately $3000-4200 \mathrm{~mL} \mu \mathrm{m} \mathrm{m}^{-2}$ day $^{-1}$ at $25^{\circ} \mathrm{C}$ and different relative humidities ${ }^{12}$ and WVTR values of around $420 \mathrm{~g} \mathrm{\mu m} \mathrm{m}^{-2}$ day $^{-1}$ at $23^{\circ} \mathrm{C}$ and $85 \% \mathrm{RH}^{13}$, while PLA shows an OTR ranging from $11000-36000 \mathrm{~mL} \mu \mathrm{m} \mathrm{m}^{-2}$ day $^{-1}$ at $23^{\circ} \mathrm{C}^{14,15}$ and a WVTR of $5250 \mathrm{~g} \mu \mathrm{m} \mathrm{m}^{-2}$ day $^{-1}$ at $23^{\circ} \mathrm{C}$ and $90 \% \mathrm{RH}^{16}$. 
In order for PLA to become an industrial alternative to PET, an even more significant reduction is therefore required.

For the specific case of PLA, the effect of crystallinity on water barrier properties in general, and on water sorption in particular, is still not fully understood, and so many different results can be found in the literature. Some reports, for instance, claim that water absorption decreases in line with increased crystallinity $^{17}$, while others report the opposite behaviour ${ }^{18}$. Finally, some papers report different behaviours in water sorption and water diffusion measurements, depending on test conditions ${ }^{19}$. In any case, despite crystallinity effects, it has been proven that improvements in the barrier properties of nanocomposites is related, at least partially, to the nanomateria| ${ }^{20}$. This is known to play an important role in mass transport phenomena by increasing the tortuous path, and thus decreasing diffusivity.

Although PLA nanocellulose composites have exhibited better performance compared to PLA/nanoclay in terms of enhanced barrier and thermomechanical properties, a combination of both nanocellulose and nanoclay within the polymer matrix would be an interesting combination that might enhance the performance of the material even further. Such a combination of nanoclay and nanocellulose fibres was investigated in preparation of thin nanoclay/nanocellulose films. In one case, addition of 20 wt\% of vermiculite to a CNF matrix led to a decrease in OTR of $86 \%$ at $50 \%$ RH and $94 \%$ at $80 \% \mathrm{RH}^{21}$, while incorporation of $50 \mathrm{wt} \%$ of $\mathrm{MNT}$ to a $\mathrm{CNF}$ matrix resulted in a five-fold reduction in OTR at $95 \% \mathrm{RH}^{22}$.

The combination of nanoclay and nanocellulose has also been investigated in polymer composites, where poly(vinyl alcohol)/nanocellulose-based composites showed increased thermomechanical properties, reduced strain at break and a decreased oxygen transmission rate above $30 \% \mathrm{RH}$ (although they also showed increased water absorption) in line with increased clay content ${ }^{23}$. The replacement of $1 \mathrm{wt} \%$ of nanoclay with $1 \mathrm{wt} \%$ of $\mathrm{CNC}$ in a PLA/montmorillonite composite containing $5 \mathrm{wt} \%$ nanoclay led to an eight-fold increase of strain at break for the composites (from $10.6 \%$ to $78.8 \%)^{24}$. In a similar fashion the addition of CNC to the composite led to enhanced water absorption and 
different water diffusion, depending on the amount of $\mathrm{CNC}$ added ${ }^{25}$. Finally, the combination of CNC and nanoclay led to improved thermomechanical properties for PLA-grafted maleic acid composites ${ }^{26}$. The present work aimed at determining possible synergistic effects as a result of combining nanocellulose and nanoclay in PLA nanocomposites, by focusing on properties that will have to be improved in order for PLA to become an industrially relevant alternative polymer for food packaging.

\section{Experimental}

\section{Materials}

Polylactic acid (Ingeo 2003D) was supplied by Natureworks (Minnesota, USA). Cellulose nanoreinforcements were extracted from sisal (Agave Sisalana, which was kindly supplied by Expor Sisal S.L) according to a previously described procedure ${ }^{11}$. The nanoclay was Cloisite 30B (C30B), which is commercially available and well described in the literature ${ }^{27-29}$. $\mathrm{NaOH}$, sulfuric acid (95\%-97\%), nitric acid (ACS reagent, 70\%), acetic acid (99\%-100\%), N, N- dimethyl formamide (98\%, ACS reagent) and dichloromethane ( $99.8 \%$ chromasolv) were purchased from Sigma Aldrich, while sodium chlorite ( $25 \%$ w/w on water) was supplied by Merck. All of the reagents were used as received.

\section{Preparation of CNF and CNC}

The acetylated cellulose nanofibre (CNF) and nanocrystalline cellulose (CNC) extraction and isolation procedures have been described elsewhere ${ }^{11}$, a summary of which can be found in the supporting information. Briefly, this method is based on grafting acetate groups onto the surface of the swollen cellulose nanofibres, in order to inhibit hydrogen bonding, thus allowing for the easy extraction and individualisation of the fibres. The protocol consists of a sequence of chemical treatments (alkali treatments, bleaching and acetylation) of the sisal fibres followed by preparing for the dispersion of nanofibres (either CNF or CNC) in DMF via magnetic stirring.

\section{Nanocomposite preparation}

A neat PLA film and two types of hybrid composites with 1 or 5 wt\% of nanocellulose in combination with 1, 3 and 5 wt\% of nanoclay (PLA/CNF/C3OB and PLA/CNC/C3OB) were prepared by mixing three premixes followed by film formation by solvent casting in Teflon moulds. A specially prepared rack 
ensured the high reproducibility of film thicknesses and uniform films. PLA/C3OB, PLA/CNF and PLA/CNC composites were used as reference materials, prepared as described elsewhere ${ }^{11}$, and a summary of the preparation procedure is included in the supporting information.

\section{A) Neat PLA reference film}

PLA (5 g) was dissolved in dichloromethane $(250 \mathrm{~mL}$ ) by magnetic stirring overnight at room temperature. The mixture was degassed in an ultrasonic bath for 5 minutes, and the PLA solution was then poured slowly into three Teflon moulds, $80 \mathrm{~mL}$ in each mould. The three moulds were covered by a 5-13 $\mu \mathrm{m}$ filter paper, kept in a Climacell climatic chamber (MMM Group) at $23^{\circ} \mathrm{C}$ for 16 hours and thereafter dried at $50^{\circ} \mathrm{C}$ in a vacuum for at least 24 hours. The prepared PLA films were $75 \mu \mathrm{m} \pm 2.3$ $\mu \mathrm{m}$ in thickness.

\section{B) $\mathrm{PLA} / \mathrm{CNF} / \mathrm{C} 30 \mathrm{~B}$ and PLA/CNC/C30B composite films}

The hybrid composites were prepared by mixing a standard solution of PLA (A), a standard dispersion of nanocellulose (for both CNF and $\mathrm{CNC}$ ) (B) and a standard dispersion of nanoclay (C). Both nanocellulose dispersions in DMF (B) were obtained directly as a result of the preparation procedure as a $0.8 \mathrm{w} / \mathrm{v} \%$ dispersion. Nanoclay dispersion (C) was achieved by stirring Cloisite C30B (2 g) in DMF $(200 \mathrm{~mL})$ for 24 hours, followed by ultrasonication at $200 \mathrm{~W}$ for 30 minutes, to obtain a stable dispersion that could be used for preparing the hybrid composites. The PLA standard solution (A) was freshly prepared prior to use by magnetically stirring PLA $(3.3 \mathrm{~g})$ in DMF $(65 \mathrm{~mL})$ vigorously at $70^{\circ} \mathrm{C}$ for 2 hours. Dispersions with a specific nanocellulose and nanoclay content were then prepared by mixing premixes A, B and C, all of which were diluted to $105 \mathrm{~mL}$ with additional DMF. The dispersions were stirred vigorously and ultrasonicated at $200 \mathrm{~W}$ for 10 minutes and finally cast into a Teflon mould $(12 \mathrm{~cm} \times 12 \mathrm{~cm})$. The solvent was then removed by drying at $80^{\circ} \mathrm{C}$ for 15 hours, which was followed by additional drying in vacuo at $50^{\circ} \mathrm{C}$ for at least 24 hours. Film thicknesses were measured at least nine different points with a digital micrometer (with an absolute error of $1 \mu \mathrm{m}$ ). It was found that all of the composites showed similar thicknesses and that each film had a small standard deviation. These differences in thicknesses between the different films can be attributed to the inherent uncertainty 
of the solvent casting procedure rather than to the effect of the nanofillers. The hybrid composites PLA/CNF/C30B showed a thickness ranging from 81 to $93 \mu \mathrm{m}$ with a standard deviation below $3.1 \mu \mathrm{m}$, while the PLA/CNC/C30B composites were prepared with a thickness ranging from 86 to $95 \mu \mathrm{m}$ with a standard deviation below $4.5 \mu \mathrm{m}$.

\section{Characterisation methods}

The dispersion of nanofillers within the polymer matrix was studied by X-Ray diffraction (XRD) (Philips $X^{\prime}$ Pert Pro diffraction system utilising a Cu-tube $(\lambda=1.542 \AA)$, operating at $40 \mathrm{kV}$ and $40 \mathrm{~mA}$, and scanning electron microscopy (SEM) (FEI Quanta 200 ESEM FEG) and transmission electron microscopy (FEI Tecnai T20 G2). The thermal properties (glass transition temperature $\left(T_{g}\right)$, melting temperature $\left(T_{m}\right)$ and degree of crystallinity $\left(X_{c}\right)$ ) of neat PLA and the nanocomposites were determined by differential scanning calorimetry (DSC) (TA DSC Q1000), using a heating rate of $10^{\circ} \mathrm{C} / \mathrm{min}$ across a range of $0-200^{\circ} \mathrm{C}$ in a heating/cooling/heating cycle. The melting enthalpy of $100 \%$ crystalline poly(Llactide) was considered to be $93 \mathrm{~J} \mathrm{~g}^{-1}$, as reported previously ${ }^{30}$. To determine $X_{c}$, the following expression was used for both the first and the second heating cycle:

$$
X c=\frac{\Delta H_{\mathrm{m}}-\Delta H_{\mathrm{C}}}{\Delta H_{0}}
$$

where $\mathrm{X}_{c}$ is the degree of crystallinity of the composite, $\Delta H_{m}$ is melting enthalpy, $\Delta H_{c}$ is crystallisation enthalpy and $93 \mathrm{~J} \mathrm{~g}^{-1}$ was considered as the melting enthalpy of $100 \%$ crystalline PLA. Isothermal crystallisation studies were performed by heating samples from RT to $200^{\circ} \mathrm{C}$ at $10^{\circ} \mathrm{C} / \mathrm{min}$, where they were stabilised for 2 minutes and thereafter cooled down to $0^{\circ} \mathrm{C}$ at $20^{\circ} \mathrm{C} / \mathrm{min}$. Next, the samples were heated at $20^{\circ} \mathrm{C} / \mathrm{min}$ until they reached $120^{\circ} \mathrm{C}$ and were then kept for 2 hours at that temperature. Finally, the samples were cooled to $0^{\circ} \mathrm{C}$ at $10^{\circ} \mathrm{C} / \mathrm{min}$ and heated until they reached $200^{\circ} \mathrm{C}$ at $10^{\circ} \mathrm{C} / \mathrm{min}$. Optical properties were measured at at least three different points using a UV-Vis spectrometer (Polar Star Omega) in the range of $200 \mathrm{~nm}-1000 \mathrm{~nm}$. The oxygen transmission rate (OTR) was measured in triplicate, using a Lyssy OPT-5000 oxygen permeability tester. OTR measurements were performed at $23^{\circ} \mathrm{C}$ at $0 \%$ and $50 \%$ of $\mathrm{RH}$, and the results were expressed in $\mathrm{mL}$ 
$\mu \mathrm{m} \mathrm{m}^{-2}$ day $^{-1}$. The water vapour transmission rate (WVTR) of the films was measured in triplicate according to the norm NF H $00-030$ at $23^{\circ} \mathrm{C}$ and $50 \%$ RH. Silica gel was used as a desiccating agent, and the cups had a specific exchange surface of $S=28.27 \mathrm{~cm}^{2}$. The mass increase in the cups, due to the water absorption of silica gel, was plotted against time, and the slope was calculated. The WVTR was calculated as shown below, where $\mathrm{n}$ is the change in mass per time, I the thickness of the film and $\mathrm{S}$ is the area of the investigated surface.

$$
W V T R=\frac{n * l}{S}
$$

One-way ANOVA analysis for calculation of the statistical significance of the OTR and WVTR data was done in Minitab 17, with statistical significance established at $p<0.05$. Mechanical properties were measured using an Instron Universal Testing Machine Model 4507 (Instron Engineering Corporation, Canton, USA) equipped with pneumatic fixtures of type I BA dumbbell-shaped samples at $2.5 \mathrm{~mm} \mathrm{~min}^{-}$

1. Five specimens $5 \times 1 \mathrm{~cm}$ in size were taken from each film. Thermomechanical properties were measured in duplicate, using DMA RSA3 (TA Instruments, USA) equipment working in tensile mode. All of the measurements were performed at a constant frequency (of $1 \mathrm{~Hz}$ ) and a strain amplitude of $0.05 \%$. The distance between the fixtures was $10 \mathrm{~mm}$. The samples were heated at $10^{\circ} \mathrm{C} / \mathrm{min}$ from room temperature until reaching $110^{\circ} \mathrm{C}$, and they were kept for 20 minutes at this temperature. Thereafter, the samples were cooled down to $25^{\circ} \mathrm{C}$ at $10^{\circ} \mathrm{C} / \mathrm{min}$ and then heated to $180^{\circ} \mathrm{C}$ at $10^{\circ} \mathrm{C} / \mathrm{min}$. The analysis was repeated to check for the reproducibility of the measurements.

\section{Results and discussion}

The dispersion of nanocellulose and nanoclay in the composites was evaluated by SEM of the crosssectional views of fractured films as shown in Figure 1. 


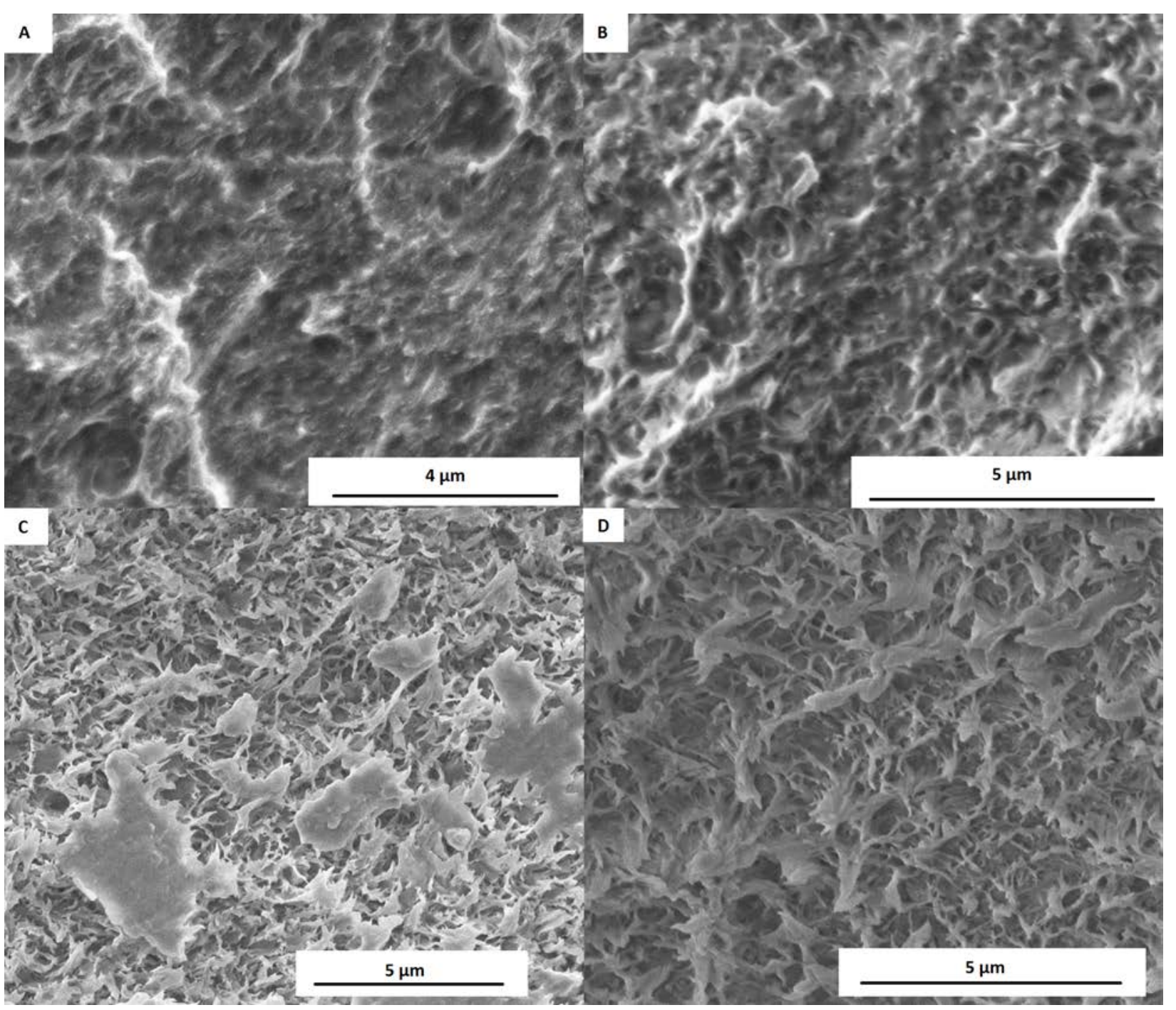

Figure 1. SEM of the cross-section of the fractured nanocomposites. A) PLA/CNF 5 wt\%/C30B 1 wt\%; B) PLA/CNC 5 wt\%/C30B 1 wt\%. C) PLA/CNF 1 wt\%/C30B 5 wt\%; D) PLA/CNC 1 wt\%/C30B 5 wt\%. 
Composites with the highest nanocellulose and the lowest C30B content ( 5 wt\% nanocellulose- 1 wt\% C30B) were chosen to evaluate the dispersion of nanocellulose, while composites with lowest nanocellulose and highest C30B content (1 wt\% nanocellulose-5 wt\% C30B) were chosen to evaluate the clay dispersion (SEMs of the cross-sectional views of the composites with $1 \mathrm{wt} \%$ nanocellulose and $1 \mathrm{wt} \%$ C30B can be seen in the supporting information, SI-Figure 1 and SI-Figure 2). The cross-sectional views of the fractured surfaces at high loading of nanocellulose (Figure $1 \mathrm{~A}$ and $1 \mathrm{~B}$ ) shows homogeneous samples without any large aggregates of nanocellulose, confirming a good dispersion of nanocellulose in the composites. Both composites with a high content of nanoclay (Figure 1C and 1D) show a significantly different fractured interface compared to the composites with a high nanocellulose loading, where the platelet structure of the nanoclays is clearly visible. The nanoclays are not as well dispersed as the nanocellulose, as can be seen from the small aggregates of up to $5 \mu \mathrm{m}$ in size for the CNF composite, whereas the CNC composite show a more homogeneous fracture surface. Considering the high content of nanoclay in these samples some aggregates would be expected on a fractured surface, as the fracture occurs at the weakest point of the film.

The effective dispersion of the nanoclay at the nanoscale was investigated further by XRD and TEM, as shown in Figure 2.
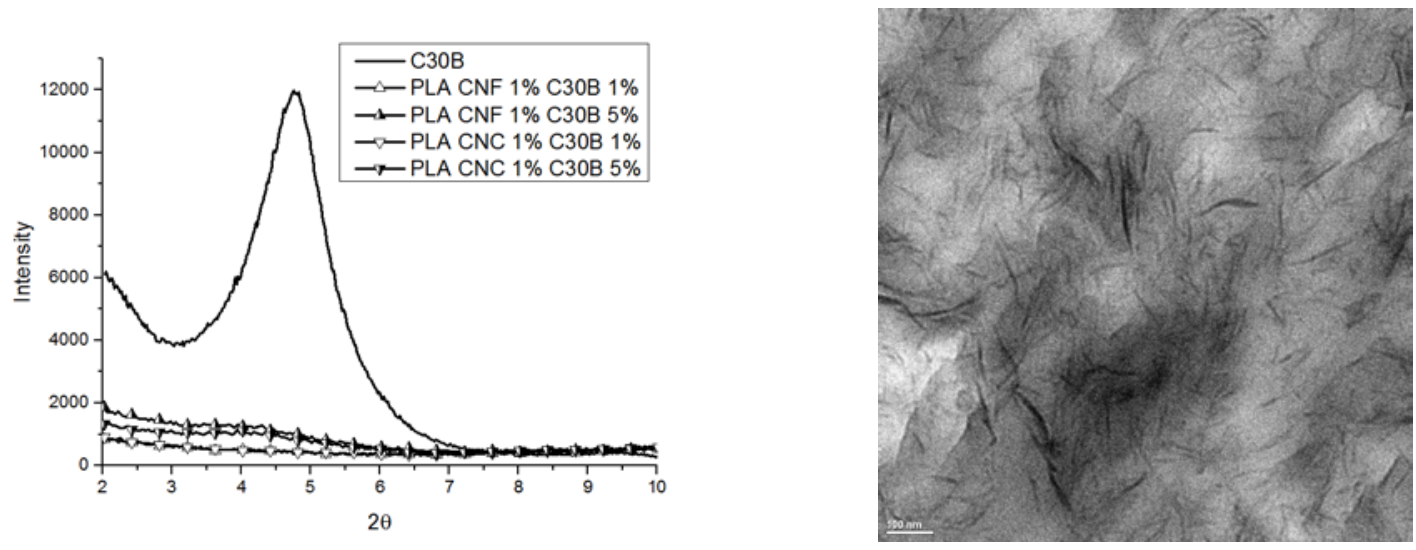

Figure 2. Left: XRD pattern of PLA/CNF/C3OB and PLA/CNC/C30B nanocomposites (\%wt) and neat C30B. Right: TEM of PLA/CNF 1 wt\%/C30B 3 wt\%. 
As can be seen from the XRD in Figure 2 (left), the neat C30B (powder) exhibits a clear diffraction peak at around $2 \theta \sim 4.5-5^{\circ}$, which corresponds to the d-spacing of the platelets within the clay. This strong diffraction peak from aggregated nanoclay is not observed for the hybrid composites, illustrating that the nanoclay platelets were separated during processing and that the nanoclay in the composites is highly exfoliated at low nanoclay loading. This could also result from dilution, however, the samples at high loading of nanoclay only show a very minor peak as an indication of the presence of small amounts of intercalated nanoclay. This was also seen from TEM of a microtomed composite (Figure 2, right). Here the nanoclay can be seen to be both intercalated (black lines) and exfoliated. In combination with the SEM micrographs from above it shows that the nanofillers are generally well dispersed with few small aggregates. In the current study the target application was food packaging materials, where barrier properties can be of great importance. As mentioned above, an important parameter in barrier properties is the crystallinity of different materials, and therefore all of the samples were evaluated by DSC, as shown in Figure 3.

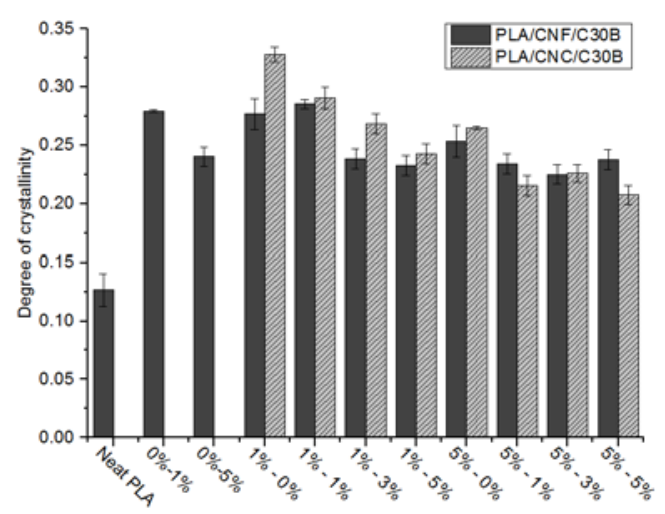

Figure 3. Degree of crystallinity of the hybrid nanocomposites.

It can be seen from Figure 3 that the addition of nanoclay or nanocellulose to the PLA results in a significant increase in crystallinity as expected. The crystallinity of the composites is seen to decrease with a higher loading of nanofiller. However, the crystallinities are considered similar for all the hybrid 
composites with above $1 \mathrm{wt} \%$ nanocellulose and $1 \mathrm{wt} \%$ nanoclay. Moreover, no relevant changes in $\mathrm{T}_{\mathrm{g}}$ or $T_{m}$ were observed in any of the composites.

PLA is known to exhibit slow crystallisation behaviour, which is a drawback for certain industrially relevant applications. Hence, the influence of nanofillers as nucleating agents in hybrid nanocomposites on the crystallisation kinetic was investigated. In Figure 4A, plots of heat flow versus time during isothermal crystallisation at $120^{\circ} \mathrm{C}$ of PLA, PLA/CNF $1 \%, \mathrm{PLA} / \mathrm{C} 30 \mathrm{~B} 1 \%$ and PLA/CNF1\% /C30B $1 \%$ are shown, while Figure 4B shows the same for PLA, PLA/CNC $1 \%$, PLA/C30B $1 \%$ and PLA/CNC 1\%/C30B $1 \%$.
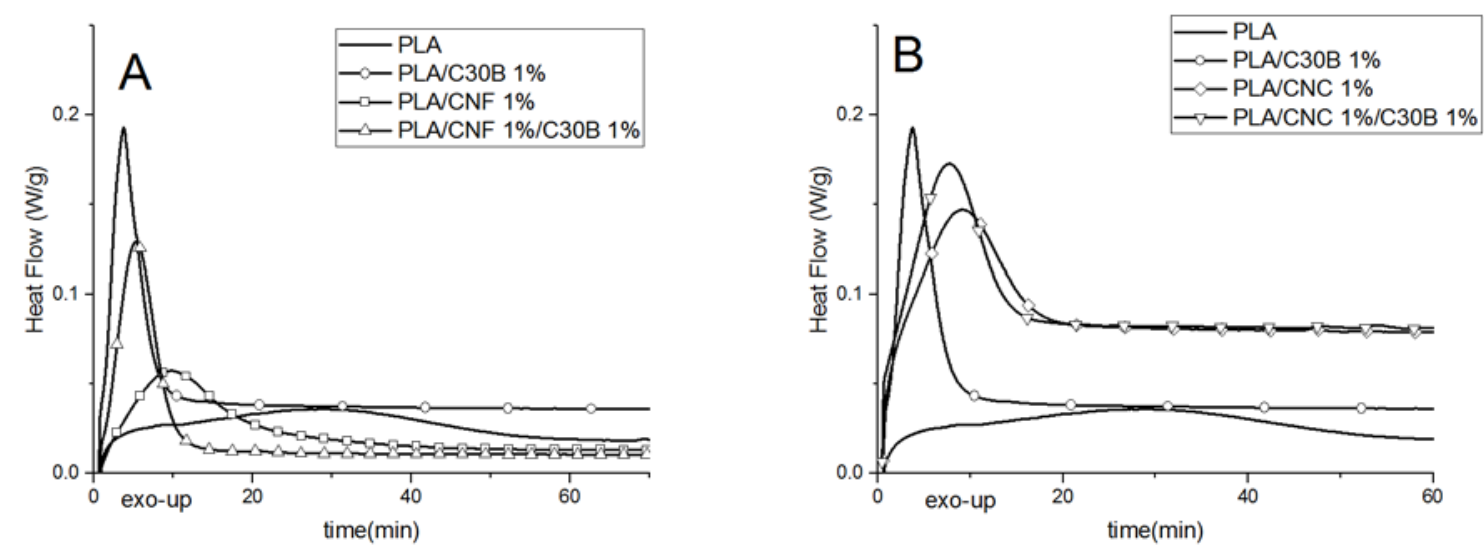

Figure 4. Heat flow versus time for the neat PLA, PLA/C30B 1\%, PLA/CNF $1 \%$ and PLA/CNF 1\%/C30B 1\% (A) and PLA, PLA/C30B 1\%, PLA/CNC 1\% and PLA/CNC 1\%/C30B 1\% (B), showing the impact on crystallisation kinetics as a result of nanocellulose and nanoclay content (\%wt).

It is clearly evident that the exothermic peak - the crystallisation peak - has shifted towards shorter crystallisation times for all of the nanocomposites, indicating that all of the nanofillers have clear nucleating agent behaviour. Nanoclay-based composites (PLA/C30B) have very fast crystallisation times compared to neat PLA, while PLA/CNF or PLA/CNC shows crystallisation behaviour between that and the neat PLA. The CNF hybrid (PLA/CNF/C30B) was found to exhibit intermediate crystallisation behaviour very similar to that of PLA/C30B, while the CNC hybrid (PLA/CNC/C3OB) showed a 
crystallisation behaviour similar to the CNC/PLA composite. Interestingly, hybrid nanocomposite, even with a low loading such as $1 \mathrm{wt} \%$ of CNF and 1 wt\% of C30B, shows a significant decrease in half crystallisation time, which is an important parameter when considering the commercial production of PLA-based packaging materials.

The oxygen transmission rate (OTR) and water vapour transmission rate (WVTR) of the nanocomposites at $23^{\circ} \mathrm{C}$ and $50 \% \mathrm{RH}$ are shown in Figure 5 .
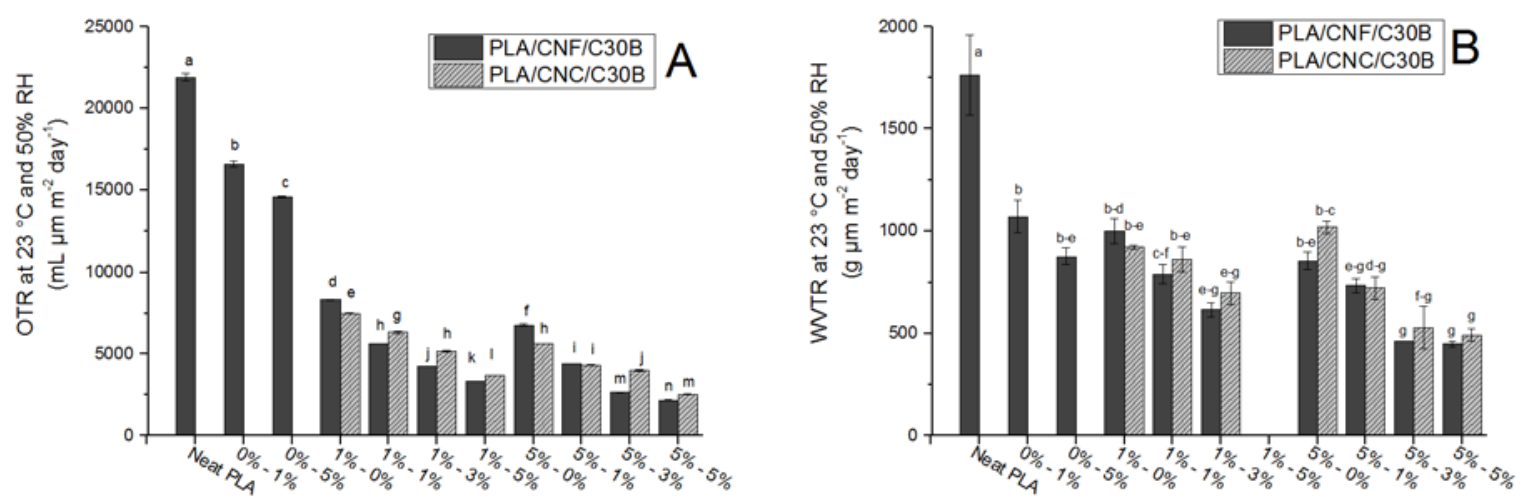

Figure 5. A) OTR and B) WVTR of the hybrid nanocomposites as a result of varied nanoclay and nanocellulose content (samples are shown as wt\% nanoclay - wt\% nanocellulose). The letters indicate significant difference (one-way ANOVA; $p<0.05$ ) between the composites.

In Figure 5 it is clear that there is a very significant decrease in the permeability of the nanocomposites compared to neat PLA. The OTR at $50 \% \mathrm{RH}$ is representative of composites measured at both $0 \%$ and $50 \% \mathrm{RH}$ (similar data for $0 \% \mathrm{RH}$ can be found in the supporting information). All of the hybrid composites show a statistically different OTR, which was reduced by $74.8 \%$ at the lowest loading of $1 \mathrm{wt} \%$ nanoclay and $1 \mathrm{wt} \%$ nanocellulose. Increasing the content of nanocellulose at $1 \mathrm{wt} \%$ nanoclay decreased oxygen permeability by $80.3 \%$ at $5 \mathrm{wt} \% \mathrm{CNF} / 1 \mathrm{wt} \%$ C30B compared to PLA, while increasing the nanoclay content to $5 \mathrm{wt} \%$ ( $1 \mathrm{wt} \% \mathrm{CNF}, 5 \mathrm{wt} \%$ C30B) resulted in a decrease in OTR of $85.0 \%$, with the highest loading ( $5 \mathrm{wt} \%$ C30B, $5 \mathrm{wt} \% \mathrm{CNF}$ ) ultimately reducing the overall OTR by $90.2 \%$ compared to PLA.

Similarly, the WVTR of the composites was also reduced significantly compared to PLA, as shown in Figure 5B. Composites at high $\mathrm{C} 30 \mathrm{~B}$ and low $\mathrm{CNF} / \mathrm{CNC}$ loading are very fragile, which unfortunately 
made it impossible to measure the WVTR for the $1 \mathrm{wt} \%$ C30B and $5 \mathrm{wt} \%$ nanocellulose composites. The WVTR was decreased by $57-76 \%$ compared to PLA, however, there is not a statistical difference in the WVTR between the different hybrid composites. It is evident that the addition of nanoclay produces a synergistic effect in combination with the nanocellulose, whereby particular nanocomposites with $5 \mathrm{wt} \%$ of C3OB and $5 \mathrm{wt} \%$ of CNF show a reduction of $90 \%$ in the OTR and $76 \%$ of the WVTR when compared to neat PLA, while already at lower amount of fillers such as $1 \mathrm{wt} \%$ C30B and $1 \mathrm{wt} \% \mathrm{CNF}$, for instance, the hybrids show a significant reduction of $74 \%$ in OTR and $57 \%$ in WVTR. This makes hybrid nanocomposites very promising for food packaging applications.

However, such large nanofiller additions, especially of nanoclay, have a significant impact on mechanical properties, as shown in Table 1.

As shown in Table 1, composites with high nanoclay content are highly brittle and show a significant reduction in elongation at break. This effect is directly correlated to nanoclay content in the composites and was also observed for the reference PLA/C30B nanocomposites as well, which were very difficult to handle. Adding nanocellulose to the composites has the effect of reducing their brittleness and enabling the handling of thin films. Especially the low loading of clay was found to provide a good combination of mechanical and barrier properties.

Although the addition of nanoclay reduces mechanical properties at room temperature, there is a significant reinforcement effect at high temperatures, as shown by the dynamic mechanical analysis (DMA) shown in Figure 6.

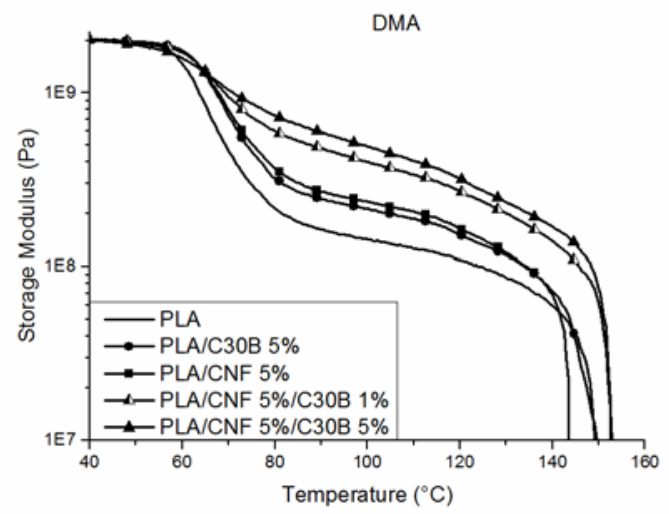


Figure 6. DMA of neat PLA and hybrid nanocomposites (\%wt).

It is well known that large surface areas of nanomaterials generally result in improved creep properties at high temperatures. The increased stability of hybrid composite films at higher temperatures is seen clearly for both the $\mathrm{C} 30 \mathrm{~B}$ and the CNF composites. Hybrid composites exhibit increased thermal stability even at low nanoclay loading, whereas increasing the content of nanoclay even further appears to increase stability only moderately. This again illustrates that the two types of nanomaterials appear to result in a synergistic effect in terms of thermal stability and a reduction in creep at higher temperature. This is expected to be a result of combining a fibrous and a platelet type of nanomaterial, where it is speculated that the nanocellulose creates a strong percolated network, whereas the platelet structure results in maintained integrity across larger areas of the film.

This added thermal stability can also be observed on the actual films after solvent casting from DMF at $80^{\circ} \mathrm{C}$, as shown in Figure 7.

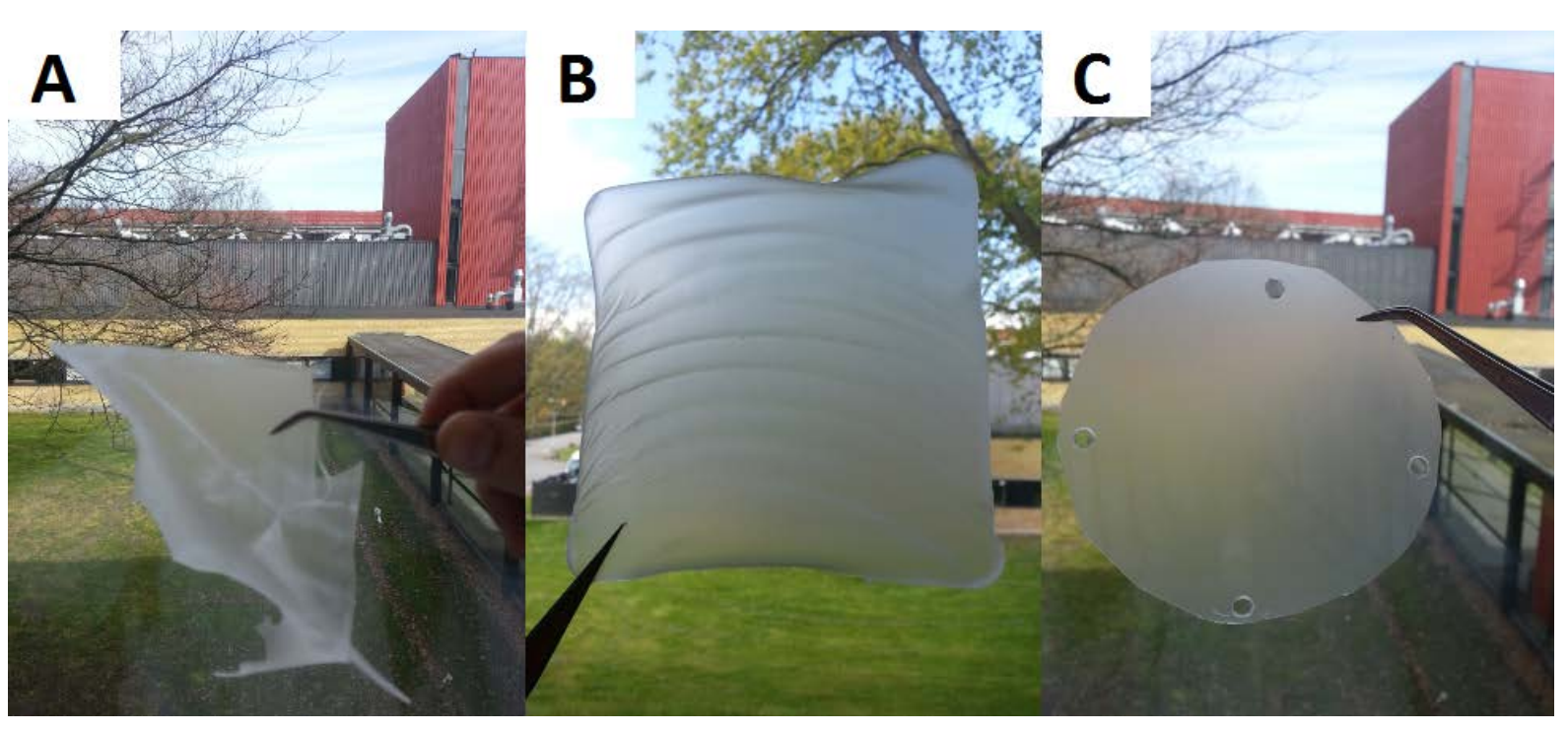

Figure 7. A) PLA, B) PLA/CNF $5 \%$ and C) PLA/CNF/C30B $1 \%-1 \%$ films after solvent casting from DMF at $80^{\circ} \mathrm{C}$.

Here, it is clear that PLA cannot resist the drying process at higher temperatures. Conversely, the PLA/CNF 5 wt\% nanocomposite can maintain film shape, although it is not completely flat. Finally, the 
hybrid PLA/CNF1\%/C30B1\% results in a completely flat film prepared in the exact same conditions, proving the synergy and the reinforcing effect on the nanocomposites.

The transparency of the films can be controlled to some extent by choosing thermal conditions during the crystallisation process. It is well known that solvent casting results in the formation of different crystalline morphologies compared to what would be expected from an industrially relevant processing method such as melt compounding. Low temperature processing, below $100^{\circ} \mathrm{C}$, as used in the solvent casting procedure, induces mainly $\alpha^{\prime}$ crystalline morphology, while processing at a higher temperature usually leads to an $\alpha$ polymorph ${ }^{31}$. Furthermore, a more significantly amorphous rigid region is also ascribed to lower processing temperatures ${ }^{32}$. The influence of processing conditions on the transmittance of the films was evaluated by UV-vis spectroscopy, as shown in Figure 8.
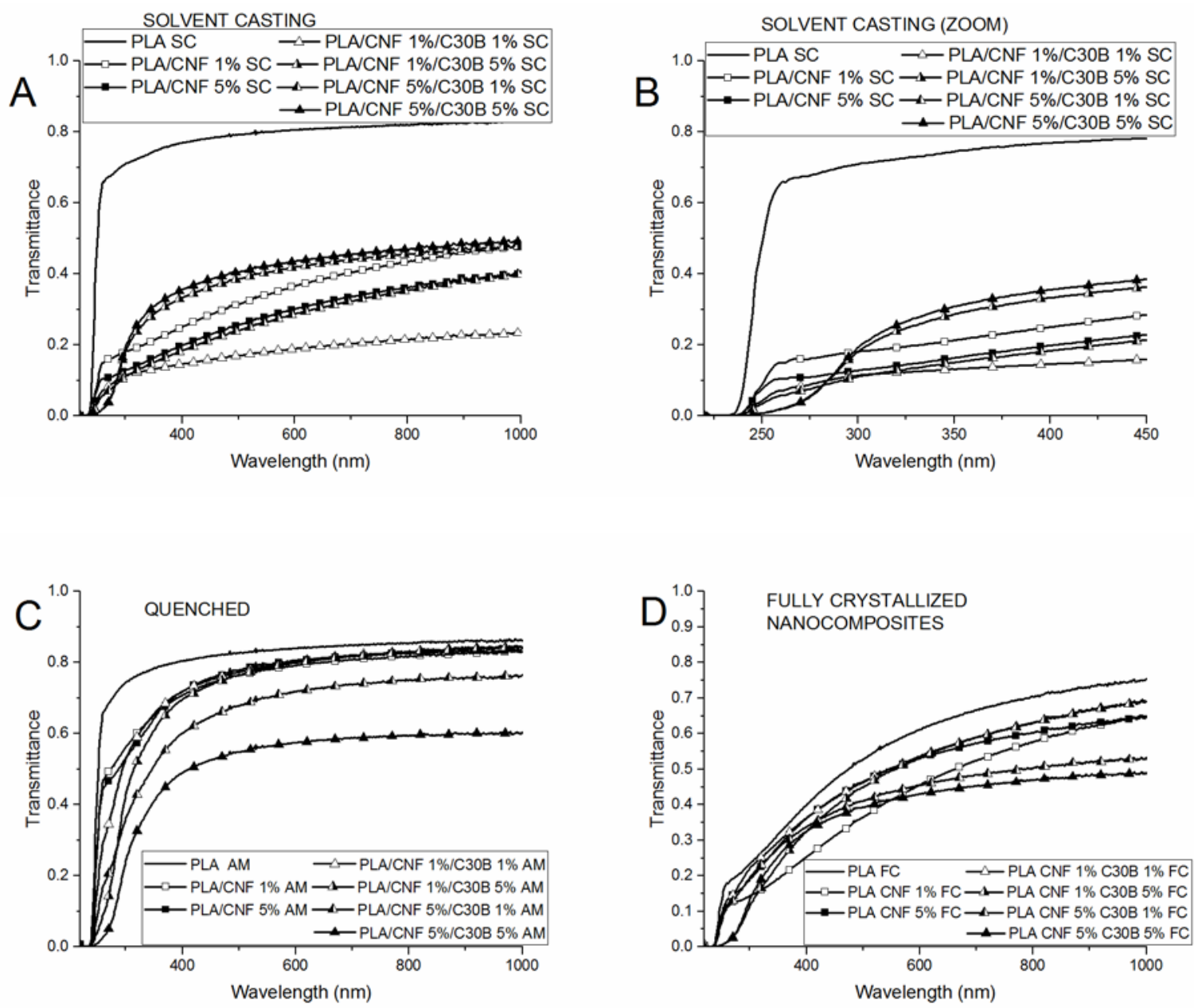
Figure 8. UV-Vis transmittance of the nanocomposites at different crystalline morphologies. A and B showing solvent-cast composites, while $\mathrm{C}$ shows the quenched samples having the lowest degree of crystallinity and $D$ the fully crystallised composites.

A quenching procedure, employed to achieve amorphous composites, followed by an isothermal crystallisation procedure at $120^{\circ} \mathrm{C}$ was performed and evaluated by UV-vis spectroscopy. There was a large difference between the composites prepared by solvent casting and the thermally treated examples. However, in general, it can be seen that most of the nanocomposites have a transmittance similar to the neat PLA when they have similar crystalline morphology. The only exception is nanocomposites with $5 \mathrm{wt} \%$ of CNF and 5 wt\% of C30B, which showed reduced transparency. Finally, it can be observed that the nanocomposites with $5 \mathrm{wt} \%$ of C30B showed significantly reduced transparency in the UV range, which is a desired property for food packaging applications. The thermally treated samples, however, were found to have small defects in the films, thereby preventing further testing for permeability. A deeper evaluation of the effect of crystallinity, amorphous regions and the amount and type of nanofiller on the water vapour barrier properties of the composites with a Quartz Spring Microbalance is currently being investigated.

\section{Conclusion}

Novel hybrid composites showing synergistic behaviour, following the combination of PLA, nanocellulose and nanoclay, were prepared. The hybrid materials showed improved properties in terms of crystallisation kinetics, maintained optical transmission as well as improved thermal and mechanical stability. It was established that the hybrid composites have significantly improved barrier properties, where the hybrid containing $5 \mathrm{wt} \%$ of C $30 \mathrm{~B}$ and $5 \mathrm{wt} \%$ of CNF showed the highest decrease in OTR of $90 \%$ and a decrease of $76 \%$ in the WVTR. Furthermore, the addition of even small quantities of nanoclay, such as $1 \mathrm{wt} \%$ of $\mathrm{C} 30 \mathrm{~B}$ and $1 \mathrm{wt} \%$ of $\mathrm{CNF}$, for example, was seen to lead to a significant decrease of $74 \%$ in the OTR and $57 \%$ in the WVTR, whilst also maintaining good thermal stability, optical properties and sufficiently good mechanical stability. Hybrid composites were identified as a very promising approach for improving PLA barrier properties and enabling the use of PLA for food 
packaging applications. Before such composites can be applied for food packaging it is of course essential to investigate the effects of having nanomaterials in close contact with foods and identify any adverse effects of disposing of such materials.

\section{Acknowledgements}

The author is thankful to the FP7 - People - 2011, ITN Marie Curie International Training Network (ITN) for their financial support and to COST Action FP1003 for funding an STSM, to Lars Schulte for the support on the SEM and to Francis Clegg and Christopher Breen for the XRD. LGP2 is part of the LabEx Tec 21 (Investissements d'Avenir - grant agreement nANR-11-LABX-0030) and of the Énergies du Futur and PolyNat Carnot Institutes (Investissements d'Avenir - grant agreements nANR-11-CARN007-01 and ANR-11-CARN-030-01).

\section{References}

1. Nampoothiri KM, Nair N, John R. An overview of the recent developments in polylactide (PLA) research. Bioresour Technol 101:8493-501 (2010).

2. Rasal RM, Janorkar AV, Hirt DE. Poly(lactic acid) modifications. Prog Polym Sci 35:338-356 (2010).

3. Sengupta R, Chakraborty S, Bandyopadhyay S, et al. A Short Review on Rubber/Clay Nanocomposites With Emphasis on Mechanical Properties. Engineering 47:21-25 (2007).

4. Bogoeva-Gaceva G, Avella M, Malinconico M, et al. Natural fiber eco-composites. Polym Compos 28:98-107 (2007).

5. Li L, Ding S, Zhou C. Preparation and degradation of PLA/Chitosan composite materials. J App/ Polym Sci 91:274-277 (2004).

6. Najafi N, Heuzey MC, Carreau PJ. Polylactide (PLA)-clay nanocomposites prepared by melt compounding in the presence of a chain extender. Compos Sci Technol 72:608-615 (2012).

7. Chen G, Kim H, Park BH, et al. Controlled Functionalization of Multiwalled Carbon Nanotubes with Various Molecular-Weight Poly(L-lactic acid). J Chem Phys B 109:22237-22243 (2005). 
8. Iwatake A, Nogi M, Yano H. Cellulose nanofiber-reinforced polylactic acid. Compos Sci Technol 68:2103-2106 (2008).

9. Aulin C, Karabulut E, Tran A, et al. Transparent nanocellulosic multilayer thin films on polylactic acid with tunable gas barrier properties. ACS Appl Mater Interfaces 5:7352-7359 (2013).

10. Eichhorn S, Dufresne A. Review: current international research into cellulose nanofibres and nanocomposites. J Mater Sci 45:1-33 (2010).

11. Trifol J, Plackett D, Sillard C, et al. A comparison of partially acetylated nanocellulose, nanocrystalline cellulose, and nanoclay as fillers for high-performance polylactide nanocomposites. J Appl Polym Sci 133:1-11 (2016).

12. Johansson F, Leufvén A. Influence of sorbed vegetable oil and relative humidity on the oxygen transmission rate through various polymer packaging films. Packag Technol Sci 7:275-281 (1994).

13. Hoffmann M. Inorganic-Organic Polymers with Barrier Properties for Water Vapor, Oxygen and Flavors. J Sol-Gel Sci Technol 2:141-146 (1998).

14. Chang J, An YU, Sur GS. Poly(lactic acid) nanocomposites with various organoclays. I. Thermomechanical properties, morphology, and gas permeability. J Polym Sci Part B Polym Phys 41:94-103 (2003).

15. Yuniarto K, Welt BA, Purwanto A, et al. Effect of Plasticizer on Oxygen Permeability of Cast Polylactic acid (PLA) Films Determined using Dynamic Accumulation Method. J Appl Packag Res 6:51-56 (2014).

16. Tsuji $\mathrm{H}$, Okino R, Daimon $\mathrm{H}$, et al. Water vapor permeability of poly(lactide)s: Effects of molecular characteristics and crystallinity. J Appl Polym Sci 99:2245-2252 (2006).

17. Cairncross RA, Becker JG, Ramaswamy S, et al. Moisture Sorption, Transport, and Hydrolytic 
Degradation in Polylactide. Appl Biochem Biotechnol 131:774-785 (2006).

18. Koo D, Du A, Palmese GR, et al. Moisture management of polylactides: The effect of heat treatment. Polymer 53:1115-1123 (2012).

19. Siparsky GL, Voorhees KJ, Dorgan JR, et al. Water transport in polylactic acid (PLA), PLA/polycaprolactone copolymers, and PLA/polyethylene glycol blends. J Environ Polym Degrad 5:125-136 (1997).

20. Picard E, Espuche E, Fulchiron R. Effect of an organo-modified montmorillonite on PLA crystallization and gas barrier properties. App/ Clay Sci 53:58-65 (2011).

21. Aulin C, Salazar-Alvarez G, Lindström T. High strength, flexible and transparent nanofibrillated cellulose-nanoclay biohybrid films with tunable oxygen and water vapor permeability. Nanoscale 4:6622-6628 (2012).

22. Liu A, Walther A, Ikkala O, et al. Clay nanopaper with tough cellulose nanofiber matrix for fire retardancy and gas barrier functions. Biomacromolecules 12:633-41 (2011).

23. Spoljaric S, Salminen A, Dang Luong N, et al. Nanofibrillated cellulose, poly(vinyl alcohol), montmorillonite clay hybrid nanocomposites with superior barrier and thermomechanical properties. Polym Compos 35:1117-1131 (2013).

24. Arjmandi R, Hassan A, Mohamad Haafiz MK, et al. Partial replacement effect of montmorillonite with cellulose nanowhiskers on polylactic acid nanocomposites. Int J Biol Macromol 81:91-99 (2015).

25. Arjmandi R, Hassan A, Haafiz MKM, et al. Effect of hydrolysed cellulose nanowhiskers on properties of montmorillonite/polylactic acid nanocomposites. Int J Biol Macromol 82:9981010 (2016).

26. Hong J, Kim DS. Preparation and physical properties of polylactide/cellulose nanowhisker/nanoclay composites. Polym Compos 34:293-298 (2013). 
27. Mohapatra AK, Mohanty S, Nayak SK. Poly(lactic acid) and layered silicate nanocomposites prepared by melt mixing: Thermomechanical and morphological properties. Polym Compos 33:2095-2104 (2012).

28. Katiyar V, Gerds N, Koch CB, et al. Melt processing of poly(L-lactic acid) in the presence of organomodified anionic or cationic clays. J Appl Polym Sci 122:112-125 (2011).

29. Rhim JW, Hong SI, Ha CS. Tensile, water vapor barrier and antimicrobial properties of PLA/nanoclay composite films. LWT - Food Sci Technol 42:612-617 (2009).

30. Sinha Ray S, Okamoto M. Biodegradable polylactide and its nanocomposites: Opening a new dimension for plastics and composites. Macromol Rapid Commun 24:815-840 (2003).

31. Zhang J, Tashiro K, Tsuji H, et al. Disorder-to-order phase transition and multiple melting behavior of poly (I-lactide) investigated by simultaneous measurements of WAXD and DSC. Macromolecules 41:1352-1357 (2008).

32. Righetti MC, Tombari E. Crystalline, mobile amorphous and rigid amorphous fractions in poly(L-lactic acid) by TMDSC. Thermochim Acta 522:118-127 (2011).

Table 1 Mechanical properties of all hybrid composites, determined by tensile testing ${ }^{a}$.

\begin{tabular}{|c|c|c|c|c|c|c|c|c|}
\hline \multicolumn{9}{|c|}{ CNF/C30B } \\
\hline & & PLA & $1 \%-1 \%$ & \multirow{3}{*}{$\begin{array}{c}1 \%-3 \% \\
2.7+/- \\
0.2\end{array}$} & \multirow{3}{*}{$\begin{array}{c}1 \%-5 \% \\
2.7+/- \\
0.1\end{array}$} & \multirow{3}{*}{$\begin{array}{c}5 \%-1 \% \\
2.9+/- \\
0.1\end{array}$} & \multirow{3}{*}{$\begin{array}{c}5 \%-3 \% \\
3.1+/- \\
0.1\end{array}$} & \multirow{3}{*}{$\begin{array}{c}5 \%-5 \% \\
3.1+/- \\
0.2\end{array}$} \\
\hline & & & $2.5+/-$ & & & & & \\
\hline$E$ & (GPa) & $2.4+/-0.3$ & 0.2 & & & & & \\
\hline$\sigma$ & (MPa) & $55+/-2$ & $53+/-2$ & $43+/-1$ & $18+/-5$ & $51+/-3$ & $27+/-2$ & $16+/-3$ \\
\hline & & & $2.9+/-$ & $2.2+/-$ & $0.9+/-$ & $2.6+/-$ & $1.6+/-$ & $0.7+/-$ \\
\hline$\varepsilon$ & $\%$ & $3.9+/-0.2$ & 0.1 & 0.3 & 0.4 & 0.1 & 1.2 & 0.1 \\
\hline \multicolumn{9}{|c|}{$\mathrm{CNC} / \mathrm{C30B}$} \\
\hline & & PLA & $1 \%-1 \%$ & $1 \%-3 \%$ & $1 \%-5 \%$ & $5 \%-1 \%$ & $5 \%-3 \%$ & $5 \%-5 \%$ \\
\hline
\end{tabular}




\begin{tabular}{ccccccccc}
$\mathrm{E}$ & $(\mathrm{GPa})$ & $2.4+/-0.3$ & $0.2+/-$ & $2.3+/-$ & $2.2+/-$ & $2.6+/-$ & $2.7+/-$ & $3.0+/-$ \\
$\sigma$ & $(\mathrm{MPa})$ & $55+/-2$ & $47+/-6$ & $40+/-2$ & $18+/-5$ & $49+/-2$ & $34+/-1$ & $25+/-3$ \\
& & & $3.1+/-$ & $2.4+/-$ & $1.2+/-$ & $3.0+/-$ & $1.6+/-$ & $1.1+/-$ \\
$\varepsilon$ & $\%$ & $3.9+/-0.2$ & 0.2 & 0.2 & 0.3 & 0.5 & 0.3 & 0.2 \\
\hline
\end{tabular}

a) $E$ is the Young's modulus in GPa, $\varepsilon$ strain at breaking point and $\sigma$ stress at breaking in $\mathrm{MPa}$. 